\title{
CORRELATION BETWEEN LYMPHOCYTE-MONOCYTE RATIO AND CYTOKINES IN CHRONIC INFLAMMATION IN RATS TREATED WITH ALLOGENEIC MESENCHYMAL STEM CELLS
}

\author{
Petrik N., Shevchenko O. \\ Kharkiv National Medical University, Kharkiv, Ukraine \\ https://doi.org/10.35339/ic.7.3.109-117
}

\begin{abstract}
A chronic inflammatory process is a pathological condition characterized by an ongoing active inflammatory response and tissue destruction. Many studies show that chronic inflammation can play a severe role in various age-related diseases, including diabetes, cardiovascular, and autoimmune diseases. One of the important but poorly studied factors affecting the regulation of chronic inflammation is regulatory activity of MSCs. In this regard, the study of mesenchymal stem cells preventing chronic inflammation in the experiment is an important area of modern pathology.

On the one hand, increased cytokines, such as $\alpha$-TNF, IL 6, and CRP, are reliable tools in diagnosis of different inflammatory processes, especially chronic inflammation. On the other hand, we need a more straightforward and not so expensive criterion for this purpose, for instance, a common total blood count and LMR. For the first time, we investigated how trustworthy can be LMR and how possible to use it in chronic inflammation in rats to achieve prognostic goals.

This study investigated the correlation between $\alpha$-TNF, IL-6, and CRP with LMR in rats' plasma in groups with chronic carrageenan inflammation and chronic inflammation with local injection of MSCs into the affected area. The study involved 132 adult male rats (180-220 g), which were divided into groups. The inflammation model was chronic aseptic myositis caused by an intramuscular injection of $10 \mathrm{mg} \lambda$-carrageenan (Sigma-Aldrich $\mathrm{GmbH}$ ). Our experimental groups of rats were treated with MSCs (the injection into the inflamed site) in the amount of 12 million cells once. Blood sampling was performed from 6 hours to 28 days. We calculated our results using Statistica (data analysis software) version 13. For comparison, we used oneway ANOVA, Turkey's post hoc test, where $\mathrm{p}<0.05$ was considered statistically significant. In our experiment, the correlation between levels of $\alpha$-TNF, IL-6, and CRP with lymphocytemonocyte ratio in rats was described for the first time, demonstrating the suppression of chronic inflammation through MSCs.

Keywords: chronic inflammation, mesenchymal stem cells, lymphocyte-monocyte rate, tumor necrosis factor-alpha; interleukin 6; C-reactive protein.
\end{abstract}

\footnotetext{
Abbreviations:

MSCs - mesenchymal stem cells;

Car $-\lambda$-carrageenan;

WBC - white blood cells count;

LMR - lymphocyte-monocyte ratio;

$\alpha-\mathrm{TNF}$ - tumor necrosis factor alpha;

IL-6 - interleukin 6;

CRP - C-reactive protein.
}

\section{Introduction}

A chronic inflammatory process is a pathological condition characterized by an

Corresponding Author:

Nataliia Petryk MD, PhD student

of the Department of Pathophysiology.

Kharkiv National Medical University, Ukraine.

E-mail: perfectgynecologist@gmail.com ongoing active inflammatory response and tissue destruction. A significant number of immune cells, including macrophages, neutrophils, and eosinophils, are involved directly or through the production of inflammatory cytokines in the pathogenesis of chronic inflammation [1].

It is well known from the literature that there is a general concept according to which chronic inflammation is the leading cause of cancer and aging processes [2]. Moreover, many studies show that chronic inflammation can play a role in various age-related diseases, including diabetes, cardiovascular, and autoimmune diseases [3]. One of the important but poorly studied factors affecting the regulation of chronic inflammation is the regulatory activity of MSCs. 
Human MSCs are individual progenitor cells that can be found in most vascularized body tissues. These cells have differentiation potential and are characterized by immunomodulatory and trophic activity [4].

At first, this was met with great skepticism, then the immunomodulatory ability of mesenchymal stem cells was proven and well reproduced in experiments $[5,6]$ and opened up the possibility of using mesenchymal stem cells for tissue replacement and regeneration and the treatment of immune-mediated and inflammatory diseases [7]. Thus, it was found that the implication of mesenchymal stem cells in the treatment of inflammatory diseases could give the most significant effect [8].

It is the fact that there are many works covering regenerative properties of mesenchymal stem cells [9-17], there are very few studies dedicated to the pathogenetic effect of mesenchymal stem cells on the processes of chronic inflammation $[18,19]$.

In recent years, there has been a tendency towards an increase in type 2 diabetes, obesity [20], cancer [21, 22], which are a consequence of chronic inflammation and lead to early mortality and disability.

In this regard, the study of mesenchymal stem cells preventing chronic inflammation in the experiment is an important area of modern pathology.

It is well known that increased cytokines, such as $\alpha$-TNF, IL 6 , and CRP, are reliable tools in diagnosis of different inflammatory processes, especially chronic inflammation [23]. (Fig. 1) Still, on the other hand, we need a more straightforward and not so expensive criterion for this purpose, for instance, a common total blood count and LMR

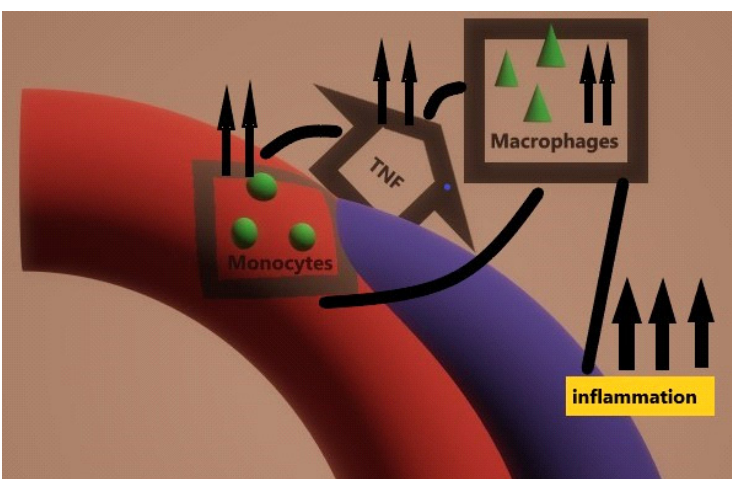

Fig. 1. Scheme of the relationship between TNF, monocytes, and macrophages. The positive feedback of TNF on monocytes for their functioning and then transforming into macrophages, which leads to enhancing the course of chronic inflammation
[24]. LMR is an important prognostic marker of endothelial dysfunction and inflammation. Low LMR correlates with worsening recovery and is probably a prognostic criterion for developing diseases associated with chronic inflammation.

In our study, we investigated how trustworthy can be LMR, and if it is possible to use it in chronic inflammation in rats to achieve prognostic goals.

\section{Purposes, subjects and methods:}

\subsection{Purpose}

This study investigated the correlation between $\alpha$-TNF, IL-6, and CRP with LMR in rats' plasma in groups with chronic carrageenan inflammation and chronic inflammation with local injection of MSCs into the affected area.

\subsection{Subjects \& Methods}

The study involved 132 adult male rats (180$220 \mathrm{~g}$ ), which were divided into groups. The inflammation model was chronic aseptic myositis caused by intramuscular injection of $10 \mathrm{mg}$ $\lambda$-carrageenan (Sigma-Aldrich $\mathrm{GmbH}$ ) into the right hip [25].

The studies were carried out under the national "General Ethical Principles for Animal Research" (Ukraine, 2001) [26] (Strasbourg, 18.03.1986 p.), the Declaration of Helsinki, (1964-2000), the charter of the Ukrainian Association for Bioethics and GLP (1992) and used the minimum acceptable for statistical processing and obtaining reliable results, the current number of animals ( 6 per group). The animals were sacrificed with inhalation of high concentrations of carbon dioxide (CO2), followed by decapitation.

\section{Isolations of MSCS}

We isolated MSCs from the rat femur bone marrow using the standard method [27-30]. The bone marrow was withdrawn from the femur epiphyses then washed with Hanks solution (Biowest, France). The cells were centrifuged to pellet (1000 rpm, 10 min). Mononuclear cells (Fig. 2)

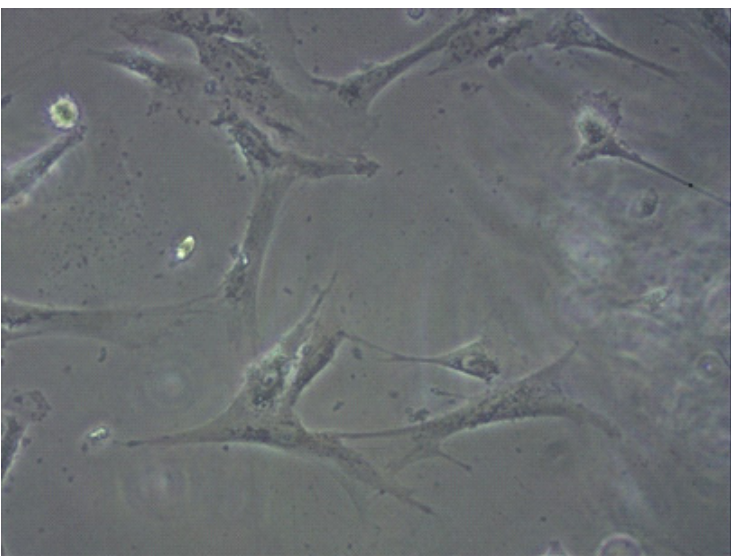

Fig. 2. MSCs of the femoral bone marrow of male rats 
were obtained by centrifugation in a FicollHypaque gradient (density $1.077 \mathrm{~g} / \mathrm{ml}$ ) (Sigma, USA) at $400 \mathrm{~g}$ for $25 \mathrm{~min}$ and washed twice with Hanks solution (Biowest, France). After that, the cells were resuspended in physiological saline at a concentration of $1.0 \times 106$ in $1 \mathrm{ml}[31,32]$. We measured the total number of cells with a cytometer by staining with $0.2 \%$ trypan blue solution (Janssen Chemica, Belgium). The structure of MSCs was investigated using a phasecontrast microscope; we studied cell cycles by flow cytometry. The immunocytochemical method studied MSCs phenotype.

Primary cultured MSCs were oval, fusiform, or polygonal, adhered to a plastic surface within 24 hours, and reached $90 \%$ confluence within eight days. After cleaning and breeding, they were equally long, spindle-shaped, and transmitted every five days. The adhesion rate was complete within 24 hours. Flow cytometry showed that $80 \%$ of fourth-generation MSC cells were in the G0 phase. Immunocytochemical analysis showed that MSCs were positive for CD29, CD105, CD166, VLA-4, and P-selectin, but negative for CD34 and CD45.

Experiment and blood collection in rats

In the experiment, sixty rats had edema of the right thigh due to the intramuscular injection of $\lambda$-carrageenan. The other sixty rats were simultaneously injected not only with carrageenan but also with a suspension of MSCs. The control group consisted of six intact rats without intervention and six rats that were injected with MSCs without inflammation.

The quantity of MSCs was 2 million cells in $0.4 \mathrm{ml}$ per animal. There were ten terms in the experiment. For each assignment, we analyzed six rats with inflammation of carrageenan and six rats with inflammation plus MSCs. Animals were sacrificed under anesthesia after 6 hours on days $1,2,3,5,7,10,14,21,28$. Blood samples were obtained by cardiac puncture. The blood smears were performed immediately (Fig. 3), and some blood was collected to the sterile tubes containing an anticoagulant (EDTA) for total blood count. Empty sterile tubes were used for plasma preparation. A blood clot appeared in 25-30 min, then the tubes were placed in a centrifuge and processed at $3000 \mathrm{rpm}$ for $10 \mathrm{~min}$. Plasma was obtained and sent to the freezer $\left(-20^{\circ} \mathrm{C}\right)$.

Determination of $\alpha-T N F, I L-6$, and CRP

Plasma $\alpha$-TNF, IL-6, and CRP levels were measured using an enzyme-linked immunosorbent assay kit (Sigma-Aldrich $\mathrm{GmbH}$ ) for quantitative measurement of target markers in biological fluids. We used ELISA for rat TNF-?, ELISA for rat IL-6, ELISA for rat CRP (C-reactive protein).

\section{The lymphocytes monocytes ratio}

LMR in rats was calculated in the same way as in humans [33-40]. We took the absolute number of lymphocytes and divided them by the complete number of monocytes. As a result, we found a positive trend of LMR increasing. On day 21, LMR was significantly higher in the group of animals with chronic inflammation and MSCs.

\section{Statistics}

All calculations were performed using Statistica (data analysis software) version 13. For comparison, we used one-way ANOVA, Turkey's test, where $\mathrm{p}<0.05$ was considered statistically significant.

\section{Conflict of interests}

The authors of the article declare no conflict of interest.

\section{Results \& Discussion}

Elevated levels of proinflammatory cytokines accompany the majority of chronic inflammatory conditions. There are several therapeutic options for lowering these levels. These include monoclonal antibodies and cytokine receptor blockers, immunosuppressants, and non-steroidal antiinflammatory drugs. None of these drugs are entirely safe or effective. Consequently, there is still a need to develop new approaches that can
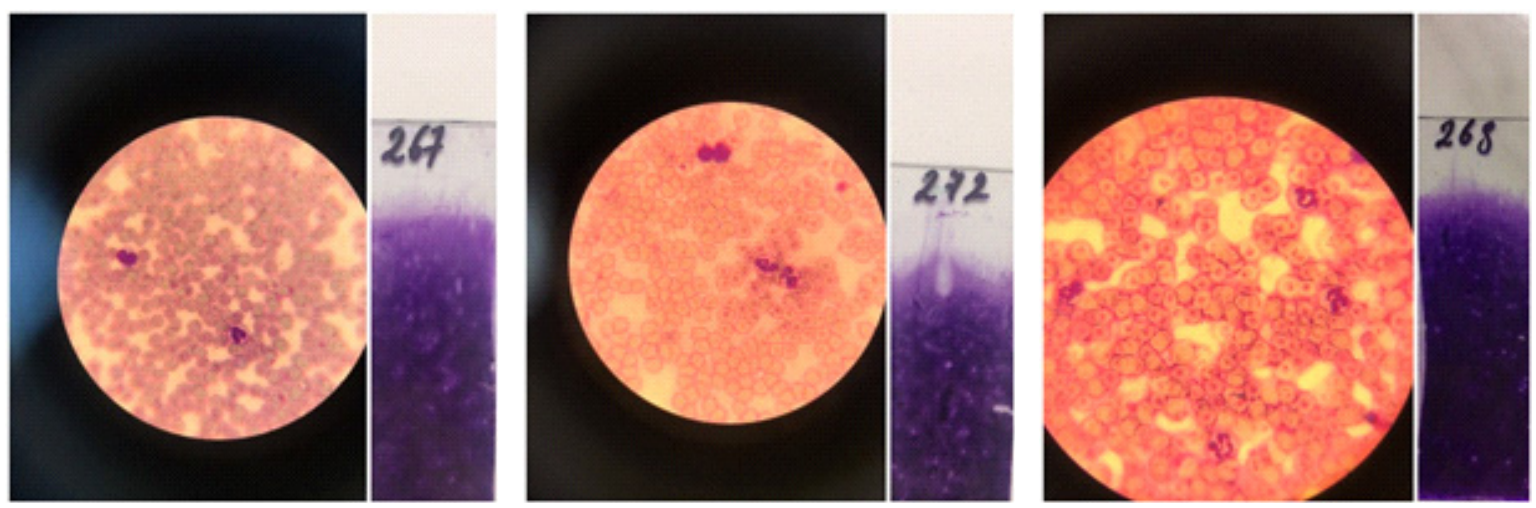

Fig. 3. Peripheral blood smears of rats 
target other pathogenetic mechanisms. MSCs may be one such approach for decreasing the production of cytokines.

The main objective of our study showed that the introduction of bone marrow MSCs in the area of chronic inflammation led to a significant decrease in proinflammatory cytokines, such as IL-6, TNF $\alpha$, and CRP, in the plasma of animals of the inflammatory group plus MSCs (Fig. 5-7). recovers on the 5 th day. Since the $7^{\text {th }}$ day, it was always superior during the next days with a peak on the $21^{\text {st }}$ day (Fig. 4)

The immunosuppressive activity of MSCs can explain these results. MSCs can support many types of immune cells, including B cells, T cells, dendritic cells (DC), natural killer cells (NK), neutrophils, and macrophages [41]. Interaction mechanisms are based on cell-cell contact,

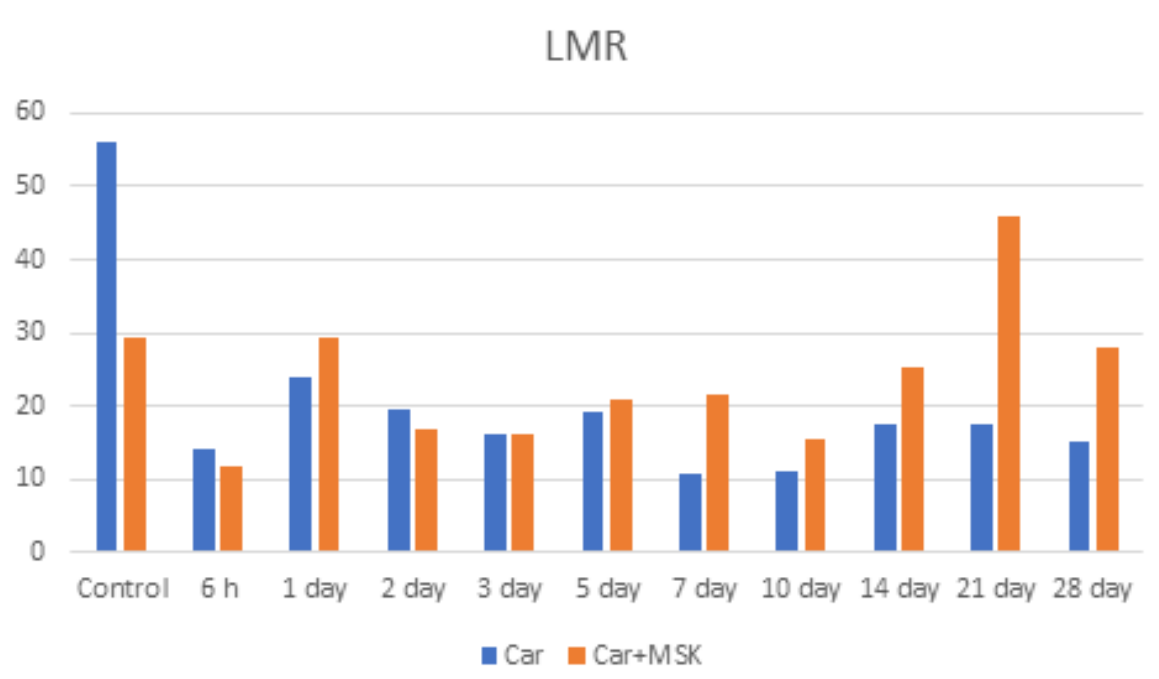

Fig. 4. Lymphocyte-monocyte ratio. The red line is the level of LMR in groups with inflammation treated with MSCs. Blue line - lymphocyte-monocyte rate in the usual course of inflammation

This decrease was statistically significant. LMR was significantly higher in animals with chronic inflammation and MSCs on the 1st day, then working in conjunction with the secretion of soluble immune factors to induce MSC-regulated immunosuppression [42]. These specific modulators

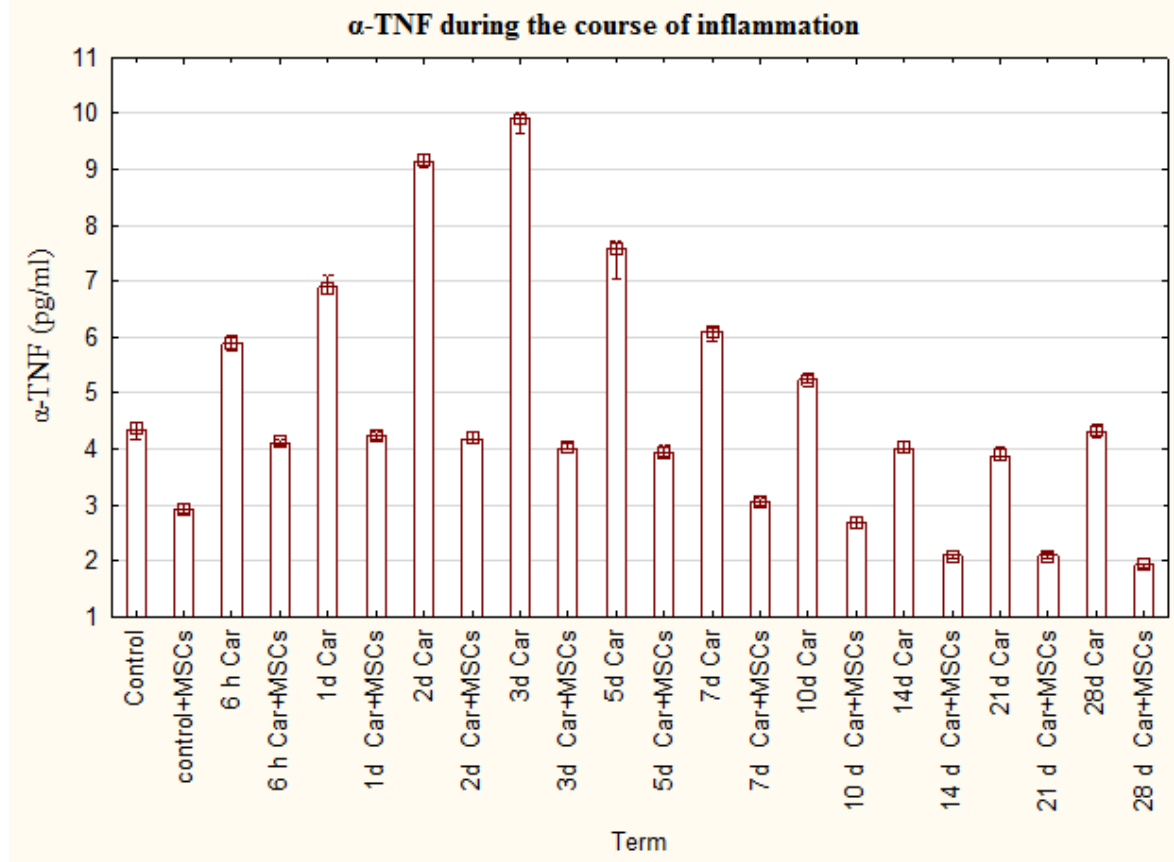

Fig. 5. The levels of $\alpha$-TNF (natural course of inflammation and inflammation treated with MSCs) 


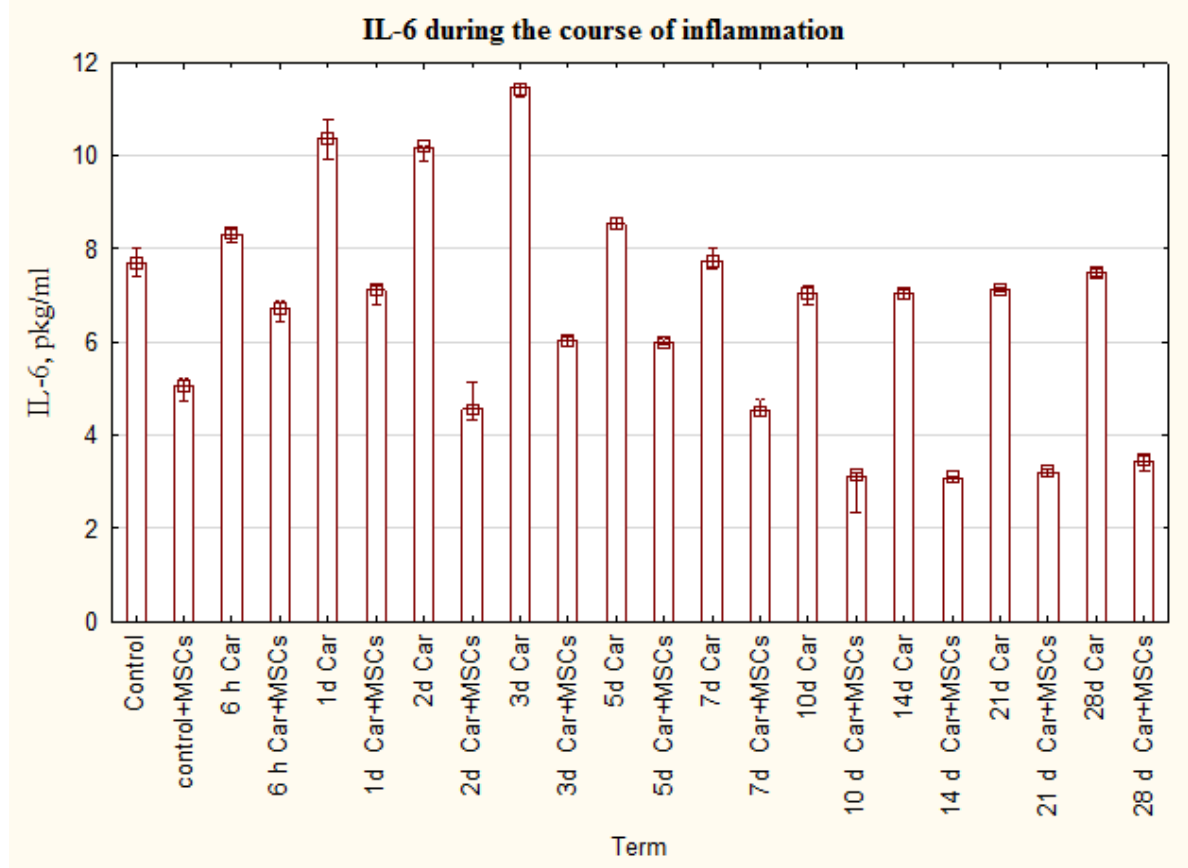

Fig. 6. The levels of IL-6 (natural course of inflammation and inflammation treated with MSCs)

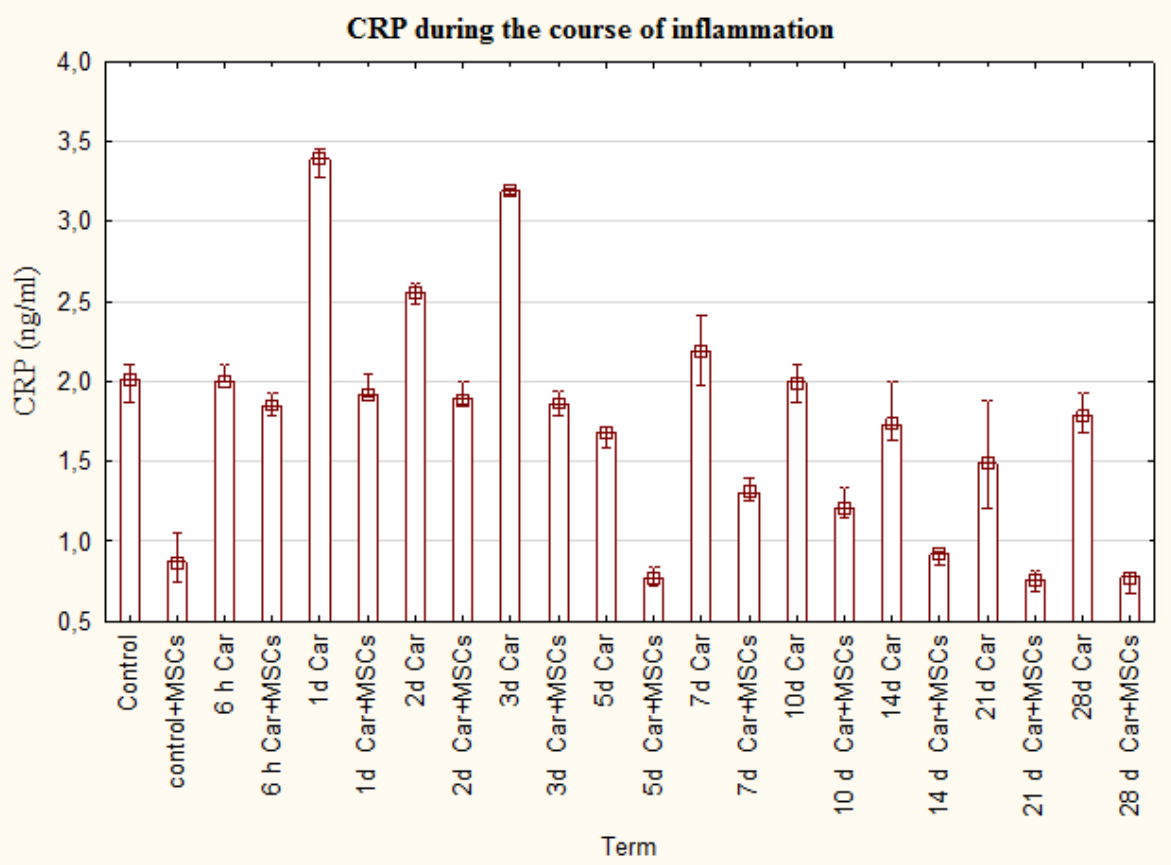

Fig. 7. The levels of CRP (natural course of inflammation and inflammation treated with MSCs)

include immunomodulatory factors, cytokines, growth factors, modulate inflammatory responses, and immune balance profiles. MSCs can also regulate the inflammatory process and repair damaged cells and tissues by attaching to the inflammation area [43]. The integration of MSCs with inflammatory processes enhances and suppress the immune response and depends on the general state of the immune system [44]. Surprisingly, MSCs modulate immunosuppression only when they are initially stimulated by inflammatory cytokines such as tumor necrosis factor (TNF) and interleukin- (IL-) 1 [45]. MSCs respond to inflammatory cytokines and produce immunoregulatory secretors that mediate the process of inflammation $[46,47]$. 
It is crucial to admit that, even though we used allogeneic bone marrow MSCs, there was a significant decrease in the cytokines in the plasma of animals in the control group plus MSCs compared to the control group. Thus, this can be explained by the immunomodulatory ability of MSCs [48]. Such a significant decrease in proinflammatory cytokines may indicate the nonimmunogenic properties of allogeneic MSCs. This fact may be necessary in cases where it is impossible to obtain autologous MSCs.

Even though the understanding of the mechanisms of immunomodulation based on MSCs remains incomplete, the growing volume of data prompts further studies of the properties of MSCs and their practical application. We believe that our research could help develop pathogenetic treatments for chronic inflammatory and autoimmune diseases that do not have side effects.

\section{Conclusions}

Our study shows that MSCs are able to mitigate severity of the inflammatory response. MSKs also showed good immunomodulatory properties. The LMR can be reliably used as an isolated immunological measurement in chronic inflammation or along with IL-6, $\alpha$-TNF, highly sensitive C-reactive protein. It can be reliable to use LMR in the course of chronic inflammation in rats to achieve prognostic goals.

There is an excellent potential for further research into preventing chronic inflammation using MSCs, including clinical investigations. We see a great potential in the future study of stem cells as immunomodulatory and anti-inflammatory agents for increasing the quality and longevity of human life.

Acknowledgment: This project was supported by Kharkiv National Medical University, Kharkiv, Ukraine.

\section{References}

1. Oliver Soehnlein, Sabine Steffens, Andres Hidalgo, Christian Weber. (2017). Circadian Control of Inflammatory Processes in Atherosclerosis and Its Complications Arteriosclerosis. Thrombosis, and Vascular Biology. 2017; 37:1022-1028.

2. J.D. Walston (2017). The fire of chronic inflammation in older adults: etiologies, consequences, and treatments. Innovation in Aging, Volume 1, Issue suppl_1, 1 July 2017, Page 961, https://doi.org/ 10.1093/geroni/igx004.3462.

3. Bertrand Fougere, Eric Boulanger, Fati Nourhash?mi, Sophie Guyonnet, Matteo Cesari. (2016). Chronic Inflammation: Accelerator of Biological Aging. The Journals of Gerontology: Series A, Volume 72, Issue 9, September 2017, Pages 1218-1225, https://doi.org/10.1093/gerona/glw240.

4. Caplan AI, Bruder SP. (2001). Mesenchymal stem cells: building blocks for molecular medicine in the 21 st century. TRENDS in Molecular Medicine Vol.7 No.6.

5. Szyska M, Na IK. (2016). Bone Marrow GvHD after Allogeneic Hematopoietic Stem Cell Transplantation. Front Immunol. 2016 Mar 30;7:118. doi:10.3389/fimmu.2016.00118.

6. Le Blanc K, Frassoni F, Ball L, Locatelli F, Roelofs H, Lewis I, Lanino E, Sundberg B, Bernardo ME, Remberger M, Dini G, Egeler RM, Bacigalupo A, Fibbe W, Ringden O. (2008). Lancet. 10;371(9624):1579-86. doi: 10.1016/S0140-6736(08)60690-X.

7. Griffin MD, Elliman SJ, Cahill E, English K, Ceredig R, Ritter T. (2013). Concise review: adult mesenchymal stromal cell therapy for inflammatory diseases: how well are we joining the dots? Stem Cells. (10):2033-41. doi: 10.1002/stem.1452.

8. Li-Tzu Wang, Chiao-Hsuan Ting, Men-Luh Yen, Ko-Jiunn Liu, Huey-Kang Sytwu, Kenneth K. Wu and B. Linju Yen. (2016). Human mesenchymal stem cells (MSCs) for treatment towards immune- and inflammation-mediated diseases: review of current clinical trials. Journal of Biomedical Science volume 23, Article number: 76

9. Joerg D. Hoeck, Brian Biehs, Antonina V. Kurtova, Noelyn M. Kljavin, Felipe de Sousa e Melo, Bruno Alicke, Hartmut Koeppen, Zora Modrusan, Robert Piskol, Frederic J. de Sauvage. (2017). Stem cell plasticity enables hair regeneration following Lgr5+ cell loss. Nature Cell Biology volume 19, pp. 666-676.

10. Andrea J.De Micheli, Emily J.Laurilliard, Charles L.Heinke, Hiranmayi Ravichandran, Paula Fraczek, SharonSoueid-Baumgarten, Iwijn De Vlaminck, Olivier Elemento, Benjamin D.Cosgrove. (2020). SingleCell Analysis of the Muscle Stem Cell Hierarchy Identifies Heterotypic Communication Signals Involved in Skeletal Muscle Regeneration. Cell reports. Volume 30, Issue 10, Pages 3583-3595.e5.

11. Takehito Ouchi, Taneaki Nakagawa. (2020). Mesenchymal stem cell-based tissue regeneration therapies for periodontitis. Regenerative Therapy. Volume 14, Pages 72-78. 
12.Feng Zhou, Amanda B. Reed-Maldonado, Yan Tan, Huixing Yuan, Dongyi Peng, Lia Banie, Guifang Wang, Jianquan Hou, Guiting Lin, and Tom F. Lue. (2019). Development of Male External Urethral Sphincter and TissueResident Stem/Progenitor Cells in Rats. Stem Cells and Development Mary Ann Liebert, Inc. DOI: 10.1089/scd.2019.0241.

13. Stephen M.Richardson, Gauthaman Kalamegam, Peter N.Pushpara, Csaba Matta, Adnan Memic, Ali Khademhosseini, Reza Mobasheri, Fabian L.Poletti, Judith A.Hoyland, Ali Mobasheri. (2016). Mesenchymal stem cells in regenerative medicine: Focus on articular cartilage and intervertebral disc regeneration. Methods Volume 99, 15 April 2016, Pages 69-80.

14. Rebekah M. Samsonraj, Michael Raghunath, Victor Nurcombe, James H. Hui, Andre j. Van Wijnen, Simon M. Cool. (2017). Concise Review: Multifaceted Characterization of Human Mesenchymal Stem Cells for Use in Regenerative Medicine STEM CELLS Translational Medicine doi.org/10.1002/sctm.170129.

15. Somayeh Keshtkar, Negar Azarpira, Mohammad Hossein Ghahremani, (2018) Mesenchymal stem cell-derived extracellular vesicles: novel frontiers in regenerative medicine. Stem Cell Research \& Therapy 9, 63. https://doi.org/10.1186/s13287-018-0791-7.

16. Yao Fu, Lisanne Karbaat, Ling Wu, Jeroen Leijten, Sanne K. Both, Marcel Karperien. (2017). Trophic Effects of Mesenchymal Stem Cells in Tissue Regeneration. Tissue Engineering Part B: Reviews Vol. 23, No. 6. https://doi.org/10.1089/ten.teb.2016.0365.

17. Oryan A. Kamali A. · Moshiri A. Baghaban Eslaminejad M. (2017). Role of Mesenchymal Stem Cells in Bone Regenerative Medicine: What Is the Evidence? Cell Tissue Organs https://doi.org/10.1159/ 000469704.

18.Jun Guo, Guo-sheng Lin, Cui-yu Bao, Zhi-min Hu \& Ming-yan Hu. (2007). Anti-Inflammation Role for Mesenchymal Stem Cells Transplantation in Myocardial Infarction. Inflammation volume 30, pages 97-104. https://doi.org/10.1007/s10753-007-9025-3.

19. Ugne Pivoraite, Akvile Jarmalaviciute, Virginijus Tunaitis, Giedre Ramanauskaite, Aida Vaitkuviene, Vytautas Kaseta, Gene Biziuleviciene, Algirdas Venalis, Augustas Pivoriunas. (2015). Exosomes from Human Dental Pulp Stem Cells Suppress Carrageenan-Induced Acute Inflammation in Mice. Inflammation. DOI: $10.1007 /$ s10753-015-0173-6.

20.Leitner DR, Fruhbeck G, Yumuk V, et al. (2017). Obesity and Type 2 Diabetes: Two Diseases with a Need for Combined Treatment Strategies - EASO Can Lead the Way. Obes Facts.10(5):483-492. doi:10.1159/000480525.

21. Boloker, Gabrielle et al. Updated statistics of lung and bronchus cancer in United States. (2018). Journal of thoracic disease vol. 10,3 2018: 1158-1161. doi:10.21037/jtd.2018.03.15.

22. Sitarz, Robert et al. (2018). Gastric cancer: epidemiology, prevention, classification, and treatment. Cancer management and research vol. 10 239-248. 7. doi:10.2147/CMAR.S149619.

23.Petryk N, Shevchenko O. Mesenchymal Stem Cells Anti-Inflammatory Activity in Rats: Proinflammatory Cytokines. J Inflamm Res. (2020); 13:293-301

https://doi.org/10.2147/JIR.S256932

24.Petryk, N., Shevchenko, O. Anti-inflammatory Activity of Mesenchymal Stem Cells in $\lambda$-CarrageenanInduced Chronic Inflammation in Rats: Reactions of the Blood System, Leukocyte-Monocyte Ratio. Inflammation (2020). https://doi.org/10.1007/s10753-020-01262-5

25. NA Klimenko, SV Tatarko, AN Shevchenko, GI Gubina-Vakulik. (2007). Justification of the model of chronic (secondary chronic) inflammation. Eksperim. I clinich. medicine. No. 2. p. 24-28.

26.van der Meulen-Frank M., Prins JB., Waarts BL., Hofstra W. (2017) Vertebrate Animals Used for Experimental and Other Scientific Purposes: Principles and Practice for Legislation and Protection. In: Glaudemans A., Medema J., van Zanten A., Dierckx R., Ahaus C. (eds) Quality in Nuclear Medicine. Springer, Cham. https://doi.org/10.1007/978-3-319-33531-5_5

27. Sproston NR, Ashworth JJ. (2018). Role of C-Reactive Protein at Sites of Inflammation and Infection. Front Immunol. 2018; 9:754. Apr 13. doi:10.3389/fimmu.2018.00754

28. Morrison SJ, Scadden DT. The bone marrow niche for haematopoietic stem cells. Nature. (2014);505 (7483):327-334. doi:10.1038/nature12984.

29. Susanne Kern, Hermann Eichler,Johannes Stoeve,Harald Kl?ter,Karen Bieback. (2012). Comparative Analysis of Mesenchymal Stem Cells from Bone Marrow, Umbilical Cord Blood, or Adipose Tissue. Stem Cells 24(5):1294-301. 
30.Baldev R. Gulati, Rajesh Kumar, Niharika Mohanty, Pawan Kuma, Rajesh K. Somasundaram, Prem S. Yadav. (2013). Bone Morphogenetic Protein-12 Induces Tenogenic Differentiation of Mesenchymal Stem Cells Derived from Equine Amniotic Fluid. Cells Tissues Organs 2013; 198:377-389 DOI: $10.1159 / 000358231$.

31. Kassis I, Zangi L, Rivkin R, Levdansky L, Samuel S, Marx G, Gorodetsky R. (2006). Isolation of mesenchymal stem cells from G-CSF-mobilized human peripheral blood using fibrin microbeads. Bone Marrow Transplant. 2006 May; 37(10):967-76. PMID: 16670702 DOI: 10.1038/sj.bmt.1705358.

32.K. Uchida, K. Urabe, K. Naruse, M. Ujihira, K. Mabuchi, and M. Itoman. (2007). Comparison of the cytokine-induced migratory response between primary and subcultured populations of rat mesenchymal bone marrow cells. J Orthop Sci 12:484-492 DOI 10.1007/s00776-007-1159-5.

33.Lee, Jeong Soo et al. (2018). "Reference values of neutrophil-lymphocyte ratio, lymphocytemonocyte ratio, platelet-lymphocyte ratio, and mean platelet volume in healthy adults in South Korea." Medicine vol. 97,26: e11138. doi:10.1097/MD.0000000000011138.

34.Guo, Ya-Huan et al. (2017) "The clinical use of the platelet/lymphocyte ratio and lymphocyte/ monocyte ratio as prognostic predictors in colorectal cancer: a meta-analysis." Oncotarget vol. 8,12 (2017): 20011-20024. doi:10.18632/oncotarget.15311.

35.Eo, Wan Kyu et al. (2016) "The Lymphocyte-Monocyte Ratio Predicts Patient Survival and Aggressiveness of Ovarian Cancer." Journal of Cancer vol. 7,3 289-96. 29 Jan. 2016, doi:10.7150/ jca.13432.

36. SandhyaGokavarapuMDS†Li ZhenWangDDS, MD, PhD‡ZhenTianDDS, MD, PhD§Chen PingZhangDDS, MD, PhD?. (2017). Low Pretreatment Lymphocyte-Monocyte Ratio and High PlateletLymphocyte Ratio Indicate Poor Cancer Outcome in Early Tongue Cancer. Journal of Oral and Maxillofacial Surgery Volume 75, Issue 8, August 2017, Pages 1762-1774. https://doi.org/10.1016/j.joms.2016.12.023.

37. Marek Sierzega PhD, Marzena Lenart PhD, Magdalena Rutkowska PhD, Marta Surman PhD, Bozenna Mytar MSc, Andrzej Matyja PhD, Maciej Siedlar PhD \& Jan Kulig PhD. (2017). Preoperative Neutrophil-Lymphocyte and Lymphocyte-Monocyte Ratios Reflect Immune Cell Population Rearrangement in Resectable Pancreatic Cancer. Annals of Surgical Oncology volume 24, pages 808-815. https://doi.org/ 10.1245/s10434-016-5634-0.

38. Masatsune Shibutani, Kiyoshi Maeda, Hisashi Nagahara, Yasuhito Iseki, Tetsuro Ikeya, Kosei Hirakawa. (2017). Prognostic significance of the preoperative lymphocyte to monocyte ratio in patients with colorectal cancer. ONCOLOGY LETTERS 13: 1000-1006. DOI: 10.3892/ol.2016.5487.

39. Danielle Lux, Vafa Alakbarzade, Luke Bridge, Camilla N. Clark, Brian Clarke, Liqun Zhang, Usman Khan \& Anthony C. Pereira. (2020). The association of neutrophil-lymphocyte ratio and lymphocytemonocyte ratio with 3-month clinical outcome after mechanical thrombectomy following stroke. Journal of Neuroinflammation volume 17, Article number: 60. https://doi.org/10.1186/s12974-020-01739-y

40. Selma Tekin, Esin Avci, Rukiye Nar, Eylem De?irmenci, S?leyman Demir, Hande ?enol. (2019). Are Monocyte/HDL, Lymphocyte/Monocyte and Neutrophil/Lymphocyte Ratios Prognostic or Followup Markers in Ischemic Cerebrovascular Patients? J Basic Clin Health Sci 2020; 1:38-43 Journal of Basic and Clinical Health Sciences https://doi.org/10.30621/jbachs.2020.837.

41.Y. Wang, X. Chen, W. Cao, and Y. Shi, (2014) "Plasticity of mesenchymal stem cells in immunomodulation: pathological and therapeutic implications," Nature Immunology, vol. 15, no. 11, pp. 1009-1016, 2014.

42.T. J. Kean, P. Lin, A. I. Caplan, and J. E. Dennis, (2013) "MSCs: delivery routes and engraftment, cell-targeting strategies, and immune modulation," Stem Cells International, vol. 2013, Article ID 732742, 13 pages, 2013.

43.G. Ren, L. Zhang, X. Zhao et al., (2008) "Mesenchymal stem cell-mediated immunosuppression occurs via concerted action of chemokines and nitric oxide," Cell Stem Cell, vol. 2, no. 2, pp. 141-150, 2008

44.R. Anzalone, M. L. Iacono, S. Corrao et al. (2010) "New emerging potentials for human Wharton's jelly mesenchymal stem cells: immunological features and hepatocyte-like differentiative capacity," Stem Cells and Development, vol. 19, no. 4, pp. 423-438, 2010.

45.F. M. Abomaray, M. A. al Jumah, K. O. Alsaad et al., (2016) "Phenotypic and functional characterization of mesenchymal stem/multipotent stromal cells fromdecidua basalisof human term placenta," Stem Cells International, vol. 2016, Article ID 5184601, 18 pages, 2016. 
46. Nejati M, Azami Tameh A, Vahidinia Z, Atlasi M A. (2018) Mesenchymal Stem Cells Improve Ischemic Stroke Injury by Anti-Inflammatory Properties in Rat Model of Middle Cerebral Artery Occlusion, Iran Red Crescent Med J. 2018; 20(1): e55085. doi: 10.5812/ircmj.55085.

47. Nejati, M, Tameh, AA, Atlasi, MA. (2014) Role of toll?like receptors 2 and 4 in the neuroprotective effects of bone marrow-derived mesenchymal stem cells in an experimental model of ischemic stroke. $\mathrm{J}$ Cell Biochem. 2019; 120: 8053-8060. https://doi.org/10.1002/jcb.28083

48. Gao F, Chiu SM, Motan DA, et al. (2016) Mesenchymal stem cells and immunomodulation: current status and future prospects. Cell Death Dis. 2016;7(1): e2062. Published 2016 Jan 21. doi:10.1038/ cddis. 2015.327

Received: 13-Aug-2020

Accepted: 26-Sep-2020 